Proceedings of the

International Geometry Center

Vol. 9, no. 2 (2016) pp. 42-48

\title{
О поверхностях пространства Минковского со стационарными значениями кривизны грассманова образа
}

\author{
М. А. Гречнева, П. Г. Стеганцева
}

\begin{abstract}
On the surfaces in Minkowski space with the stationary values of the curvature of the Grassmann image let us consider the classes of the surfaces in Minkowski space ${ }^{1} R_{4}$ (spacelike or timelike) with the stationary values of the curvature of the Grassmannian manifold $P G(2,4)$ along the domains, which are tangential to its the Grassmann image $\Gamma^{2}$

Аннотация. В данной работе рассматриваются классы поверхностей (времениподобные и пространственноподобные) пространства Минковского ${ }^{1} R_{4}$ со стационарными значениями кривизны грассманова многообразия $P G(2,4)$ вдоль площадок, касательных к их грассманову образу $\Gamma^{2}$.
\end{abstract}

Грассмановы многообразия и грассманов образ поверхностей евклидова пространства являются классическими примерами гладких многообразий, геометрия которых хорошо изучена в работах К. Лейхтвейса, Ю. Вонга, Ю. Муто, Ю. А. Аминова, А. А. Борисенко, А. В. Горькавого и других геометров. Несмотря на большое количество публикаций, много вопросов теории грассмановых многообразий и грассманова образа остаются нерешенными. Особенно это касается теории поверхностей многомерных неевклидовых пространств. Математической моделью пространства-времени в специальной теории относительности служит точечное псевдоевклидово пространство индекса 1, которое

\footnotetext{
Ключевые слова: Пространство Минковского, грассманово многообразие, секционная кривизна.
}

УДК 514.76 
еще называют пространством Минковского. Одним из первых исследователей грассманова многообразия псевдоевклидова пространства является Т. Ханган. Также вопросами римановой геометрии грассмановых многообразий неизотропных подпространств псевдоевклидова пространства занимался И. Маазикас. Изучению внутренней и внешней геометрии грассманова многообразия посвящены работы С. Е. Козлова и Д. В. Иванова. В данной работе будем рассматривать классы поверхностей пространства Минковского, кривизна грассманова образа которых принимает экстремальные значения.

Грассмановым многообразием $P G(2,4)$ пространства Минковского ${ }^{1} R_{4}$ (с метрикой $d s^{2}=-d x_{1}^{2}+d x_{2}^{2}+d x_{3}^{2}+d x_{4}^{2}$ ) называется множество двумерных плоскостей этого пространства, проходящих через фиксированную точку $O$.

В пространстве Минковского существуют плоскости трех типов. Тип плоскости определяется парой ортогональных векторов $\bar{x}$ и $\bar{y}$. Плоскость $\sigma$ называется пространственноподобной (соотв. времениподобной или изотропной), если существуют два ортогональных вектора $\bar{x}, \bar{y} \in \sigma$ такие, что $\bar{x}^{2}>0, \bar{y}^{2}>0$ (соотв. $\bar{x}^{2}<0, \bar{y}^{2}>0$, или $\bar{x}^{2}=0, \bar{y}^{2}>0$ ). Тогда грассманово многообразие представляет собой дизъюнктное объединение трех подмногообразий

$$
P G(2,4)={ }^{S} P G(2,4) \cup{ }^{T} P G(2,4) \cup{ }^{I z} P G(2,4) .
$$

В работе [5] изучены свойства этих подмногообразий, введена гладкая структура, получены формулы для вычисления секционной кривизны.

Пусть $V^{2}$ — регулярная неизотропная поверхность пространства ${ }^{1} R_{4}$. Поставим в соответствие каждой точке поверхности $V^{2}$ плоскость, проходящую через фиксированную точку пространства ${ }^{1} R_{4}$ и параллельную нормальной плоскости $N_{x}$ поверхности в точке $x$. Получим отображение поверхности $V^{2}$ в грассманово подмногообразие ${ }^{S} P G(2,4)$ или ${ }^{T} P G(2,4)$. Грассмановым образом $\Gamma^{2}$ поверхности $V^{2}$ называют образ указанного отображения.

\section{1. СТАЦИОНАРНЫЕ ЗНАЧЕНИЯ СЕКЦИОННОЙ КРИВИЗНЫ}

В работе [2] Ю. Вонг показал, что секционная кривизна грассманова многообразия евклидова пространства принимает значения из отрезка $[0,2]$. В работах [4] и [1] исследуются поверхности, для которых кривизна грассманова многообразия вдоль площадок, касательных к грассманову образу поверхности, принимает минимальное и максимальное значения. Из работы [6] следует, что секционная кривизна подмногообразий ${ }^{S} P G(2,4)$ или ${ }^{T} P G(2,4)$ пространства ${ }^{1} R_{4}$ может принимать 
любые действительные значения. По этой причине будем рассматривать значения секционной кривизны в точках локальных экстремумов.

При стандартном плюккеровом погружении указанных подмногообразий в пространство ${ }^{3} R_{6}$ метрика их точечных образов имеет сигнатуру $(--++)[5]$. Двумерные касательные площадки $\sigma$ к каждому из подмногообразий будем задавать бивектором $\bar{\sigma}$ с координатами $\sigma^{a b}=x^{[a} y^{b]}$, где $\bar{X}=\left(x^{a}\right)$ и $\bar{Y}=\left(y^{b}\right), a, b=1, \ldots, 4$ - касательные векторы. Тогда секционная кривизна в направлении данной площадки определяется формулой [6]:

$$
\bar{K}(\sigma)=\frac{\bar{R}_{a b c d} \sigma^{a b} \sigma^{c d}}{\left(g_{a c} g_{b d}-g_{a b} g_{c d}\right) \sigma^{a b} \sigma^{c d}},
$$

где $\bar{R}_{a b c d}$ - тензор кривизны, а $g_{i j}$ - компоненты метрического тензора подмногообразий грассманова многообразия. Формула (1.1) для каждого из подмногообразий ${ }^{S} P G(2,4)$ и ${ }^{T} P G(2,4)$ имеет вид

$$
\bar{K}(\sigma)=\frac{-\left(-\sigma^{12}+\sigma^{34}\right)^{2}+\left(\sigma^{13}+\sigma^{24}\right)^{2}}{-\left(\sigma^{12}\right)^{2}+\left(\sigma^{13}\right)^{2}+\left(\sigma^{14}\right)^{2}+\left(\sigma^{23}\right)^{2}+\left(\sigma^{24}\right)^{2}-\left(\sigma^{34}\right)^{2}} .
$$

Координаты $\sigma^{a b}$ удовлетворяют условию

$$
\sigma^{12} \sigma^{34}-\sigma^{13} \sigma^{24}+\sigma^{14} \sigma^{23}=0 .
$$

Точки локальных экстремумов находятся из системы уравнений

$$
\frac{\partial \bar{K}(\sigma)}{\partial \sigma^{i j}}=0
$$

В работе [6] рассматривались грассмановы подмногообразия неизотропных подпространств псевдоевклидова $n$-пространства произвольного индекса, найдены стационарные значения их секционной кривизны. В случае пространства ${ }^{1} R_{4}$ стационарные точки и соответствующие значения секционной кривизны имеют вид:

1) $\sigma^{12}=\sigma^{34}, \sigma^{24}=-\sigma^{13}$ и $\bar{K}(\sigma)=0$;

2) $\sigma^{12}=\sigma^{34}=0, \sigma^{23}=\sigma^{14}$ и $\bar{K}(\sigma)=1$;

3) $\sigma^{13}=\sigma^{24}=0, \sigma^{23}=-\sigma^{14}$ и $\bar{K}(\sigma)=1$.

\section{2. КЛАССЫ ПОВЕРХНОСТЕЙ СО СТАЦИОНАРНЫМИ ЗНАЧЕНИЯМИ СЕКЦИОННОЙ КРИВИЗНЫ ГРАССМАНОВА ОБРАЗА}

Опишем те классы поверхностей $V^{2}$ (пространственноподобных или времениподобных) пространства ${ }^{1} R_{4}$, для которых кривизна подмногообразий ${ }^{S} P G(2,4)$ и ${ }^{T} P G(2,4)$ вдоль площадок, касательных к их грассманову образу $\Gamma^{2}$, принимает стационарные значения. 
Как и в случае евклидова пространства [3] можно показать, что касательные векторы к грассманову образу записываются в виде

$$
\begin{aligned}
& \bar{X}=\left(\frac{L_{11}^{2}}{\sqrt{g_{11}}},-\frac{L_{11}^{1}}{\sqrt{g_{11}}}, \frac{L_{12}^{2}}{\sqrt{g_{22}}},-\frac{L_{12}^{1}}{\sqrt{g_{22}}}\right), \\
& \bar{Y}=\left(\frac{L_{12}^{2}}{\sqrt{g_{11}}},-\frac{L_{12}^{1}}{\sqrt{g_{11}}}, \frac{L_{22}^{2}}{\sqrt{g_{22}}},-\frac{L_{22}^{1}}{\sqrt{g_{22}}}\right),
\end{aligned}
$$

где $L_{i j}^{k}$ - коэффициенты вторых квадратичных форм поверхности $V^{2}$. Тогда бивектор $\bar{\sigma}$ имеет координаты:

$$
\begin{aligned}
\sigma^{12} & =\frac{L_{11}^{1} L_{12}^{2}-L_{12}^{1} L_{11}^{2}}{g_{11}}, & \sigma^{13} & =\frac{L_{11}^{2} L_{22}^{2}-\left(L_{12}^{2}\right)^{2}}{\sqrt{g_{11} g_{22}}}, \\
\sigma^{14} & =\frac{L_{12}^{1} L_{12}^{2}-L_{22}^{1} L_{11}^{2}}{\sqrt{g_{11} g_{22}}}, & \sigma^{23} & =\frac{L_{12}^{1} L_{12}^{2}-L_{11}^{1} L_{22}^{2}}{\sqrt{g_{11} g_{22}}}, \\
\sigma^{24} & =\frac{L_{11}^{1} L_{22}^{1}-\left(L_{12}^{1}\right)^{2}}{\sqrt{g_{11} g_{22}}}, & \sigma^{34} & =\frac{L_{12}^{1} L_{22}^{2}-L_{22}^{1} L_{12}^{2}}{g_{22}} .
\end{aligned}
$$

Рассмотрим случай, когда секционная кривизна принимает стационарное значение, равное нулю. Имеет место

Теорема 2.1. Пусть $V^{2} \subset{ }^{1} R_{4}$ - регулярная времениподобная поверхность с невырожденным грассмановым образом $\Gamma^{2}$. Стационарное значение секционной кривизны подмногообразия ${ }^{S} P G(2,4)$ или ${ }^{T} P G(2,4)$ вдоль любой неизотропной площадки, касательной $\kappa \Gamma^{2}$, равно нулю тогда и толъко тогда, когда выполняются условия:

1) поверхность $V^{2}$ является плоским многообразием в пространстве ${ }^{1} R_{4}$

2) поверхность $V^{2}$ имеет плоскую нормальную связность, т.е. ее первая и вторые квадратичные формы одновременно приводятся $\kappa$ диагональному виду.

Доказательство. По условию теоремы грассманов образ $\Gamma^{2}$ невырожден, то есть в каждой его точке пространство касательных векторов двумерно. Согласно [6], в каждой точке грассманова многообразия существует двумерная касательная площадка, в направлении которой кривизна принимает стационарное значение $\bar{K}(\sigma)=0$. Эта площадка задается условиями $\sigma^{24}=-\sigma^{13}$ и $\sigma^{12}=\sigma^{34}$. Первое условие запишется в виде

$$
\frac{L_{11}^{1} L_{22}^{1}-\left(L_{12}^{1}\right)^{2}}{\sqrt{g_{11} g_{22}}}=-\frac{L_{11}^{2} L_{22}^{2}-\left(L_{12}^{2}\right)^{2}}{\sqrt{g_{11} g_{22}}},
$$

откуда следует, что гауссова кривизна поверхности $V^{2}$ равна нулю, так как гауссова кривизна времениподобной поверхности вычисляется по 
формуле

$$
K=\frac{L_{11}^{1} L_{22}^{1}-\left(L_{12}^{1}\right)^{2}+L_{11}^{2} L_{22}^{2}-\left(L_{12}^{2}\right)^{2}}{\sqrt{g_{11} g_{22}}} .
$$

Второе условие имеет вид

$$
\frac{L_{11}^{1} L_{12}^{2}-L_{12}^{1} L_{11}^{2}}{g_{11}}=\frac{L_{12}^{1} L_{22}^{2}-L_{22}^{1} L_{12}^{2}}{g_{22}} .
$$

Для времениподобной поверхности можно выбрать параметризацию таким образом, что $g_{11}=-g_{22}$. Тогда равенство

$$
L_{11}^{1} L_{12}^{2}-L_{12}^{1} L_{11}^{2}=L_{22}^{1} L_{12}^{2}-L_{12}^{1} L_{22}^{2}
$$

означает выполнение условия $A B=B A$, где $A, B$ - матрицы вторых квадратичных форм поверхности. А это условие выделяет поверхности с плоской нормальной связностью.

Обратно, если времениподобная поверхность с плоской нормальной связностью имеет нулевую гауссову кривизну, то секционная кривизна ее грассманова образа равна 0 и это значение является стационарным. Действительно, поскольку поверхность имеет плоскую нормальную связность, то вторые квадратичные формы можно одновременно привести к диагональному виду, т.е. $L_{12}^{1}=L_{12}^{2}=0$. Тогда координаты бивектора $\bar{\sigma}$ запишутся в виде

$$
\sigma^{12}=\sigma^{34}=0, \quad \sigma^{13}=\frac{L_{11}^{2} L_{22}^{2}}{\sqrt{g_{11} g_{22}}}, \quad \sigma^{24}=\frac{L_{11}^{1} L_{22}^{1}}{\sqrt{g_{11} g_{22}}} .
$$

Из равенства нулю гауссовой кривизны следует, что

$$
L_{11}^{2} L_{22}^{2}=-L_{11}^{1} L_{22}^{1}
$$

Тогда секционная кривизна равна 0 и достигается при условиях

$$
\sigma^{12}=\sigma^{34}, \quad \sigma^{24}=-\sigma^{13},
$$

т.е. является стационарным значением.

Далее рассмотрим случай, когда секционная кривизна принимает стационарное значение, равное единице. Имеет место

Теорема 2.2. Пусть $V^{2} \subset{ }^{1} R_{4}$ - регулярная поверхность с невырожденным грассмановым образом $\Gamma^{2}$. Стационарное значение секиионной кривизны подмногообразия ${ }^{S} P G(2,4)$ или ${ }^{T} P G(2,4)$ вдоль любой неизотропной площадки, касательной $\kappa \Gamma^{2}$, равно единище тогда и только тогда, когда поверхность $V^{2}$ является гиперповерхностью некоторого трехмерного подпространства пространства ${ }^{1} R_{4}$. 
Доказательство. Согласно [6], в каждой точке грассманова многообразия существуют две двумерные касательные площадки, в направлении каждой из которых кривизна принимает стационарное значение $\bar{K}(\sigma)=1$. Первая площадка задается условиями $\sigma^{12}=\sigma^{34}=0$ и $\sigma^{23}=\sigma^{14}$, которые имеют вид

$$
\begin{aligned}
& L_{11}^{1} L_{12}^{2}-L_{12}^{1} L_{11}^{2}=L_{12}^{1} L_{22}^{2}-L_{22}^{1} L_{12}^{2}=0 \\
& L_{12}^{1} L_{12}^{2}-L_{11}^{1} L_{22}^{2}=L_{12}^{1} L_{12}^{2}-L_{22}^{1} L_{11}^{2} .
\end{aligned}
$$

Эти условия равносильны равенствам

$$
\frac{L_{11}^{1}}{L_{11}^{2}}=\frac{L_{12}^{1}}{L_{12}^{2}}=\frac{L_{22}^{1}}{L_{22}^{2}},
$$

из которых следует, что точечная коразмерность поверхности равна 1 , то есть она является гиперповерхностью.

Вторая площадка задается условиями $\sigma^{13}=\sigma^{24}=0, \sigma^{23}=-\sigma^{14}$ или

$$
\begin{aligned}
& L_{11}^{2} L_{22}^{2}-\left(L_{12}^{2}\right)^{2}=L_{11}^{1} L_{22}^{1}-\left(L_{12}^{1}\right)^{2}=0, \\
& L_{11}^{1} L_{22}^{2}+L_{11}^{2} L_{22}^{1}=2 L_{12}^{1} L_{12}^{2} .
\end{aligned}
$$

Эти условия могут быть записаны иначе, поскольку одну из вторых квадратичных форм всегда можно привести к диагональному виду. Положим $L_{12}^{1}=0$. Тогда условия $(2.3)$ означают, что либо одна из вторых квадратичных форм тождественно равна нулю, либо они имеют вид $I I^{k}=L_{i i}^{k} d u_{i}^{2}$. В каждом из этих случаев коэффициенты вторых квадратичных форм снова пропорциональны, то есть поверхность $V^{2}$ является гиперповерхностью.

Обратно, если поверхность $V^{2}$ вкладывается в трехмерное пространство, то

$$
\frac{L_{11}^{1}}{L_{11}^{2}}=\frac{L_{12}^{1}}{L_{12}^{2}}=\frac{L_{22}^{1}}{L_{22}^{2}}=k,
$$

где $k$ - коэффициент пропорциональности. Тогда из формулы $(1.2)$ следует, что $\bar{K}(\sigma)=1$ и это значение является стационарным.

\section{ЛитерАТУРА}

[1] Y. Muto. The gauss map of submanifolds in a euclidean space. J. Math. Soc. Japan, 30(1):85-100, 1978.

[2] Y. C. Wong. Sectional curvatures of grassmann manifolds. Proc. Nat. Acad. Sci. U.S.A., 60(1):75-79, 1968.

[3] Ю. А. Аминов. Геометрия подмногообразий. К.: Наукова думка, 2002.

[4] А. А. Борисенко and Ю. А. Николаевский. О поверхностях с максимальной кривизной грассманова образа. Мат. заметки, 48(3), 1990.

[5] М. А. Гургенидзе. О погружении грассманова многообразия псевдоевклидова пространства. Pr. Inst. Mat. Nats. Akad. Nauk Ukr. Mat. Zastos., 3(3):107-114, 2006. 
[6] И. Маазикас. K римановой геометрии грассмановых многообразий неизотропных подпространств псевдоевклидова пространства. Ученые записки Тартусского университета, (342):76-82, 1974.

Поступило в редакцию 11 апреля 2016, принято к печати 25 мая 2016.

Гречнева Марина Александровна

ЗНУ, ЗАПОРОЖЬЕ, УКРАИНА

Email: mag83@list.ru

Стеганцева Полина Георгиевна

ЗНУ, ЗАПОРОЖЬЕ, УКРАИНА

Email: steg_pol@mail.ru 